\title{
editorial
}

\section{We are what we read}

M olecular biology is some 47 years of age, a relative oldie among academic disciplines, at least compared with photonics, nanoengineering and media studies. Yet we find it increasingly difficult to define what it is. In the decades since Watson and Crick's paper was published, it has gone through several, sometimes parallel incarnations that no longer suffice to define it, even collectively. As an academic journal that proudly carries the words 'molecular biology' within its namealbeit condensed into a familiar acronym -EMBO reports has a special responsibility to ensure the relevance and dynamism of our scientific discipline.

In its purest and original form, molecular biology was simply the study of the structure and function of the components of living cells at the molecular level (nowadays more commonly called structural biology). In the 1950s, after the elucidation of the structure of DNA and of the genetic code, molecular biology became synonymous with the study of informational processes inside cells. As late as 1998, during an evaluation site visit to my institute, one member of the evaluation panel could ask the question: "How can you call yourselves molecular biologists, when none of you here is studying DNA replication, transcription or translation?" He evidently hadn't read even the titles of my publications. But his question illustrates the fact that, by the turn of the millennium, molecular biology was already more than the sum of structural biology and molecular genetics. It had encroached upon, and then simply swallowed up, an entire other discipline: cell biology.

To other scientists, molecular biology had become little more than a toolkit: blotting, cloning, sequencing and later on, bioinformatics. Indeed, after I was recruited to Tampere I came up against the fervent expectation that I would somehow empower young clinicians to solve all outstanding problems in medical genetics and pathophysiology, simply by teaching them how to hold a pipette.

A decade and a half later, we are in many ways at a point where molecular biology has spread its wings so wide as to encompass virtually the whole of what is often just called 'the life sciences'. Scour the contents pages of any of today's top 20 life science journals and $90 \%$ or more of the articles will fit nicely under our banner. Look at the composition of any of the life science review panels of the National Institutes of Health or the European Research Council and virtually every member could call him or herself a molecular biologist; even if, as I can testify from personal experience, the term still cuts little ice with US immigration officers.

Perhaps there are still a few 'real' biologists out there, such as my niece Kate, who spends her summers crouching in a tent in a mosquito-infested swamp in New Brunswick, logging the number of tweets per hour of every known species of widgeon, warbler and wren. But the truth is that the 'molecular' in molecular biologist is nowadays redundant. It persists only as a subtle badge of professional credibility, to set us apart from amateur butterfly collectors.

Where does this leave a journal such as EMBO reports? If we set out to be the 'Global Annals of Everything' it is safe to predict that we will end up as the journal of nothing in particular. Indeed, some might argue that the age of traditional subject journals is already drawing to a close, and, in the not so distant future, we will wake up in a world where just three or four global science titles compete on the basis of which has the jazziest website, the wittiest editorial blogs, the most competitive openaccess charges, and the cream of highimpact papers, while in subject matter they are all the same. In a sense, the coalescence of life sciences into one amalgam under the heading of 'molecular biology' provides an opportunity for a journal such as ours to be in the vanguard of transition to this new world of scientific publishing, even if the road seems at this point more appealing than the destination.

In so doing, we should not seek merely to combine structural biology, gene expression, cell biology and cute refinements of the tool kit. The type of molecular biology that we should be promoting is one in which these almost 'philosophical' elements are applied to biological problems of any and every kind.

So, unlike some other 'broad interest' journals, we actively encourage submissions of manuscripts delving into issues in evolution, ecology, behavioural neuroscience, development, medical biotechnology, plant science, and any other supposedly specialized area. For us, the crucial criterion, apart from the fact that it obviously has to fit the definition of a short-format report, is simply whether the actual findings are of genuine interest beyond a small community of academics. We might note that many of the papers that prove to have the least general interest are precisely those that are narrowly focused on the minutiae of cell structure or the nuts and bolts of gene expression.

For me, the value in continuing to have an old-style journal such as EMBO reports, but with new-style content, is that the content is appealing to a wide audience. Our scientific reports and reviews should be interesting enough that anyone versed in the most basic arts of biochemistry, genetics and biocomputing should want to pick up an issue and read it from cover to cover. If someone wants simply to surf into a specific article about their favourite protein, among the amorphous mass of such papers in PubMed, it might as well have been published in any of hundreds of other journals or, for that matter, in the anonymous 'twitosphere'. Our journal aspires to be different: it aims to excite and arouse with reports of novel findings, built on the fundamentals of molecular biology, but applied to any topic, whether organelles or orgasms, internal medicine or exobiology.

\section{Howy Jacobs}

EMBO reports (2010) 11, 561. doi:10.1038/embor.2010.104 